\title{
Nota clínica
}

\section{Leiomiosarcoma vesical. Presentación de un nuevo caso y revisión de la literatura}

\author{
Jorge Rioja Zuazu, David Rosell Costa, Anibal Rincón Mayans, Abel Saiz Sansi, \\ Ángel Panizo Santos*, José $\mathrm{M}^{\mathrm{a}}$ Berián Polo
}

Departamento de Urología. *Departamento de Anatomía Patológica, Clínica Universitaria. Universidad de Navarra. España

\section{Resumen}

Los leiomiosarcomas de vejiga son tumores mesenquimales excepcionales. Su diagnóstico diferencial con los leiomiomas es fundamental para decidir nuestra actitud terapéutica. Presentamos un nuevo caso de leiomiosarcoma vesical con comportamiento agresivo que ha presentado múltiples recidivas, y realizamos una revisión de la literatura para establecer las pautas terapéuticas más adecuadas.

Palabras clave: Leiomiosarcoma vesical. Diagnóstico diferencial. Tratamiento.

\section{Bladder leiomiosarcoma. A new case and a review of the literatura}

\section{Abstract}

Bladder Leiomiosarcomas are exceptional mesenchimal tumours. Their differential diagnosis is basic to decide our therapeutic attitude. We present a new case of bladder leiomiosarcoma with a bad behavior and with multiple relapses, and perform a review of the literature in order to establish more properly the therapeutic attitudes.

Keywords: Bladder leiomiosarcoma. Differential diagnosis. Treatment.

$\mathrm{L}^{\infty}$ os leiomiosarcomas vesicales son los tumores malignos mesenquimales más frecuentes de la vejiga urinaria, representan menos del $1 \%$ de los tumores vesicales malignos ${ }^{1-7}$. A pesar que estos tumores son histológicamente similares a otros leiomiosarcomas con otro origen, los rasgos morfológicos predictores de recidiva o progresión metastático de los tumores de músculo liso con origen en la vejiga son desconocidos, incluso los criterios para separar tumores musculares de la vejiga entre benignos y malignos no están bien establecidos ${ }^{8}$.

Las dos series más largas publicadas son las de Martin $^{1}$ y la de Mills ${ }^{8}$, en las que se establecen los criterios de clasificación de los leiomiosarcomas en bajo y alto grado en función de las características histológicas, como son las figuras mitóticas y del pleomorfismo nuclear, con pronósticos diferentes respecto a su capacidad de recidiva y de metastatizar.

\section{CASO CLÍNICO}

Mujer de 40 años, sin antecedentes patológicos de interés, acude a la consulta por presentar durante el último mes 8-10 episodios de hematuria ma- croscópica, indolora y sin coágulos. Asimismo refiere aumento de la frecuencia miccional, escozor miccional y tenesmo vesical, por lo que ha seguido tratamiento con ciprofloxacino y Diclofenaco durante una semana sin evidenciar mejoría.

La exploración física no evidencia ningún signo de interés (trayectos uretero-renales no dolorosos, puño percusión bilateral negativa).

Se realiza ecografía que muestra una masa intravesical de 35,7 mm de diámetro máximo, con unos riñones ecográficamente normales (Fig. 1).

Se realiza cistoscopia flexible, que pone de manifiesto una gran masa bilobulada en pared posterior de aspecto infiltrante con áreas parcialmente necróticas en su superficie.

Posteriormente se realiza Tomografía computerizada (TC) tóraco-abdominal, gammagrafía ósea y Resección trans-uretral (RTU) biopsia para establecer el diagnóstico y valorar la extensión de la enfermedad. La TC muestra una masa vesical de $8,5 \mathrm{~cm}$. de diámetro máximo, bien definida sin signos de infiltración de la grasa perivesical, ni evidencia de adenopatías de tamaño significativo (Fig. 2). 


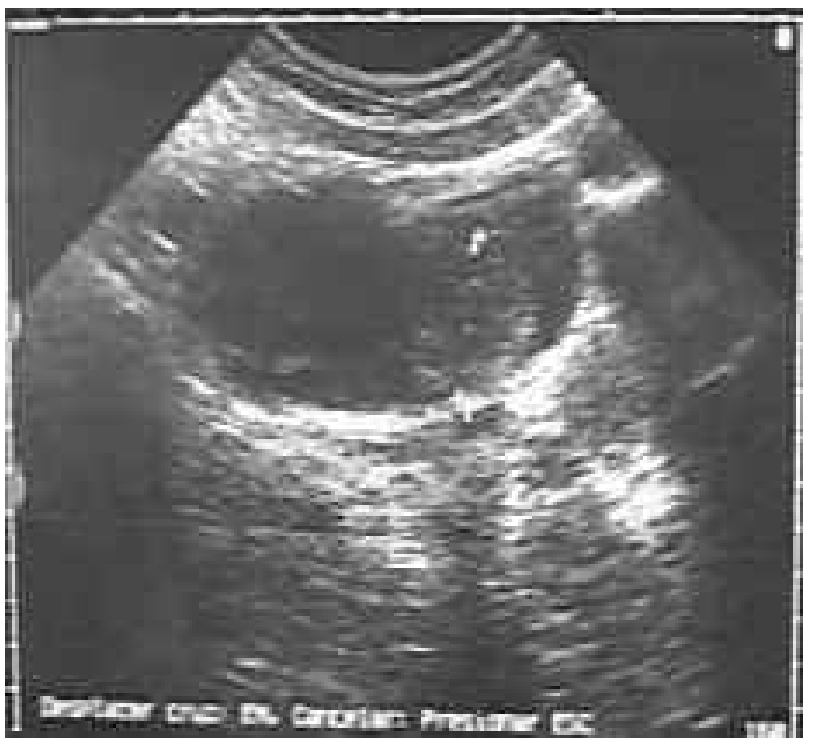

FIGURA 1. Ecografia abdominal donde se visualiza gran masa intravesical.

El estudio de extensión se completó con una gammagrafía ósea que fue normal. El estudio anatomopatológico de la RTU mostró la presencia de un tumor de aspecto mesenquimal, fusocelular, con haces largos de células fusiformes y frecuentes mitosis. El estudio inmunohistoquímico mostró una intensa inmunorreactividad con vimentina y actina muscular específica.

Con el diagnóstico de leiomiosarcoma vesical clínicamente localizado se decidió realizar exenteración pélvica anterior (cistectomía radical, histerectomía más doble anexectomía y derivación urinaria ileal).
El informe anatomopatológico de la pieza quirúrgica reveló una tumoración en la pared posterior de la vejiga de crecimiento exofítico y consistencia elástica de $8,5 \times 6 \times 4 \mathrm{~cm}$, con un pedículo de $3 \mathrm{~cm}$ de diámetro (Fig. 3).

Era un tumor mesenquimal maligno de células alargadas, de nucleolos longilíneos bordes romos y citoplasmas fibrilares. En algunas zonas se muestran figuras de mitosis (20 mitosis por 10 campos de gran aumento). El tumor se alojaba en la pared vesical, sin infiltrar el urotelio de superficie, ni la capa muscular propia vesical, presentando su origen en la capa muscular de la submucosa (Muscularis Mucosae) (Fig. 4).

Tanto el estudio de los ganglios iliacos e ilioobturatrices, así como la grasa perivesical y el resto de estructuras estudiadas, no mostraron ninguna alteración.

Tras un postoperatorio sin incidencias relevantes, se decidió completar el tratamiento con quimioterapia adyuvante que la paciente, con Adriamicina e Ifosfamida realizó en su Centro de referencia, recibiendo un total de 6 ciclos ( 1 mensual) con buena tolerancia. Se retrasó el inicio del ciclo en una ocasión por fiebre.

$\mathrm{Al}$ año de finalizar el tratamiento complementario se presentó una recidiva locorregional en una T.C. realizada durante una revisión. Por lo que se procedió a la extirpación de la lesión, con informe anatomopatológico de leiomiosarcoma de 6,5 cms. de diámetro con un borde afecto. Se decidió com-
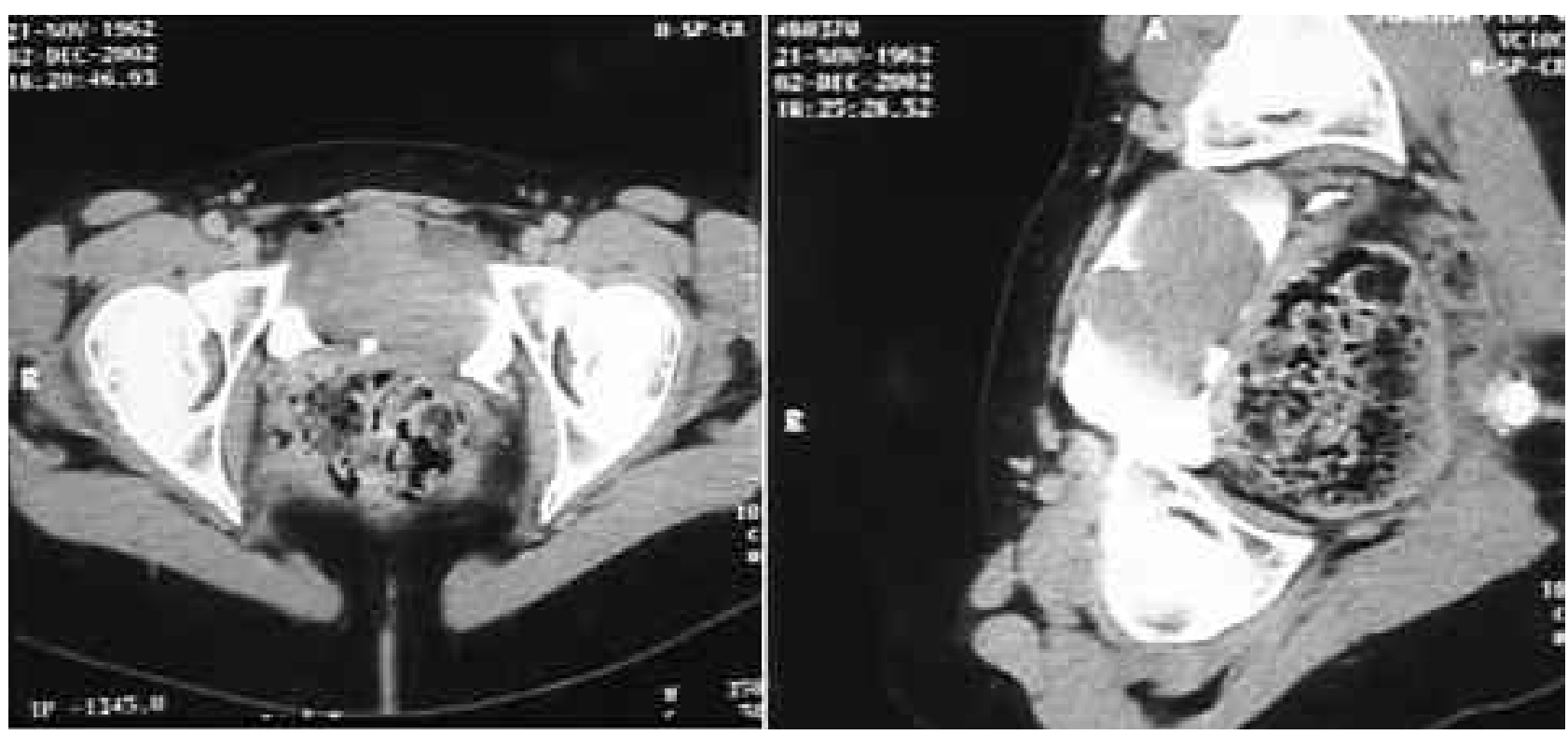

FIGURA 2. Tomografia computerizada (CT) en la que se visualiza la masa intravesical, confinada a nivel local. 


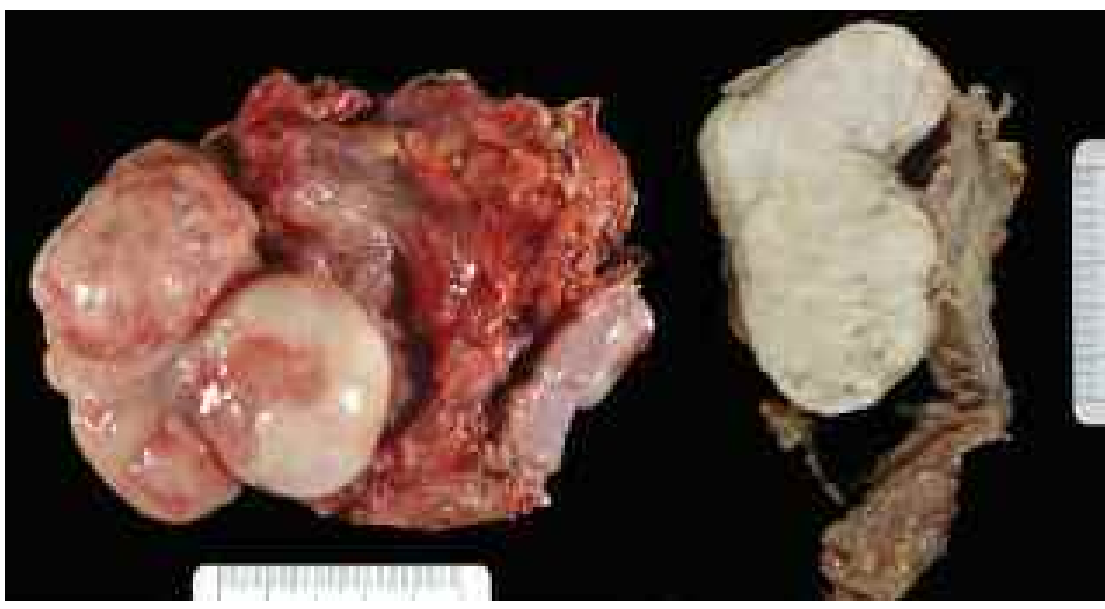

FIGURA 3. Imagen macroscópica de la pieza quirúrgica donde se observa gran tumoración pediculada y de crecimiento exocítico.

pletar el tratamiento con quicio-radioterapia adyuvante, con régimen quimioterápico basado en Ifosfamida a altas dosis $(3.520 \mathrm{mg}$ cada 24 horas por 5 días), de los cuales se ha administrado 6 ciclos, uno mensual. Una vez completados los 2 primeros ciclos de quimioterapia, recibió radioterapia externa, a razón de 1,8 Gy/día, hasta completar una dosis total de 45 Gy sobre lecho tumoral y posteriormente una sobreimpresión pélvica con 16 Gy hasta completar una dosis pélvica de 61 Gy. Posteriormente pasó a revisiones periódicas.

A los ocho meses después de haber finalizado el segundo tratamiento adyuvante, 32 meses desde el diagnóstico, la paciente vuelve a presentar una recidiva pélvica de 5 x 5,8 cm., con probable infiltración de recto-sigma y de la vejiga ileal. Se realiza rectosigmoidoscopia con punción aspiración con aguja fina, con resultado anatomopatológico de citología maligna, sarcoma fusocelular compatible con leiomiosarcoma. Se ha realizado estudio inmunohistoquímico con anticuerpos frente a Caldesmón y Miosina de músculo liso, observándose inmunorreactividad intensa de las células tumorales frente a ambos anticuerpos (Fig. 5).

Con el diagnóstico de segunda recidiva se recomendó realizar resección quirúrgica con consolidación con braquiterapia. La paciente desestimó la realización de cirugía con braquiterapia por miedo a las complicaciones de la braquiterapia, por lo que fue intervenida en otro centro, realizando exenteración pélvica posterior. En el primer control tras la cirugía, a los tres meses de la misma volvió a presentar una nueva recidiva, a nivel de la incisión, realizándose resección del mismo.

En la actualidad, y 41 meses después del diagnóstico inicial, la paciente se encuentra asintomática, en controles periódicos y sin evidencia de enfermedad.

\section{DISCUSIÓN}

Los tumores no epiteliales de la vejiga, representan del 1 al $5 \%$ de todos los tumores vesicales ${ }^{9}$. Entre los tumores de origen mesenquimal, los sarcomas vesicales son los más frecuentes, representando el $2 \%$ de todos los tumores vesicales ${ }^{1,10}$. Los

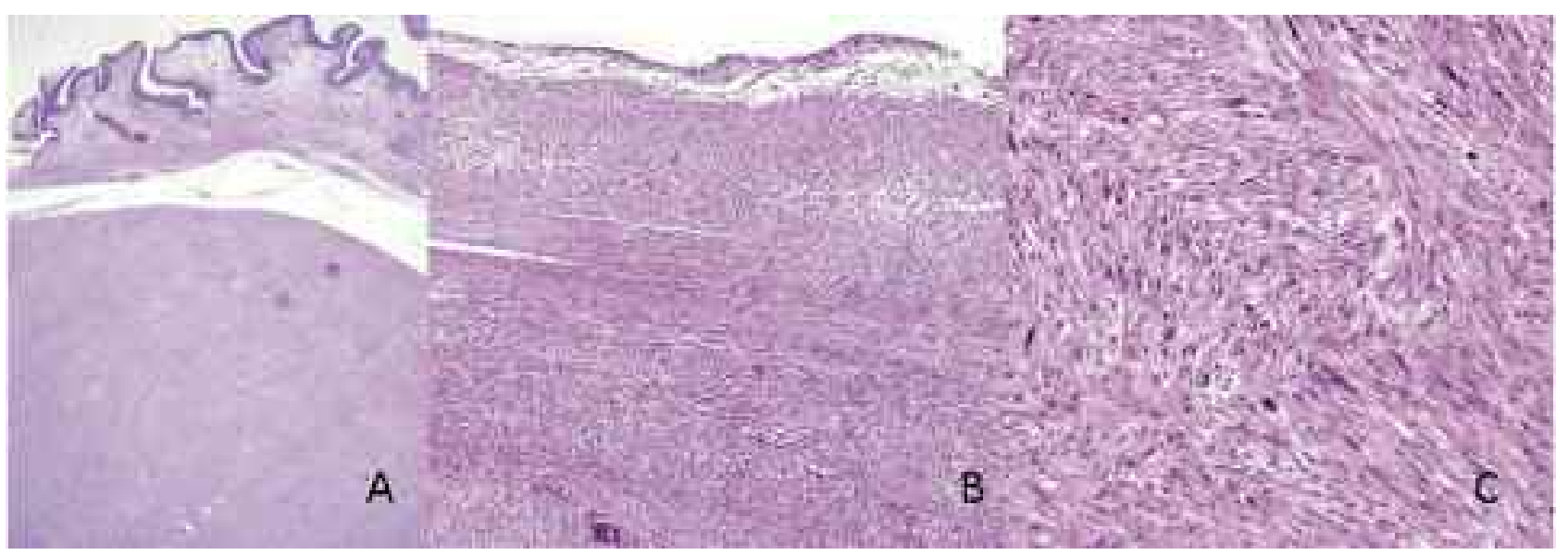

FIGURA 4. Imágenes microscópicas donde se observa la ausencia de infiltración de la capa muscular propia. A) Se visualiza la capa de mucosa perfectamente conservada, B) Imagen con mayor aumento en la que se visualiza su origen a nivel de la capa muscular de la submucosa, C) Imagen de mayor aumento donde se ven células alargadas con nucléolos longilineos y figuras de mitosis. 


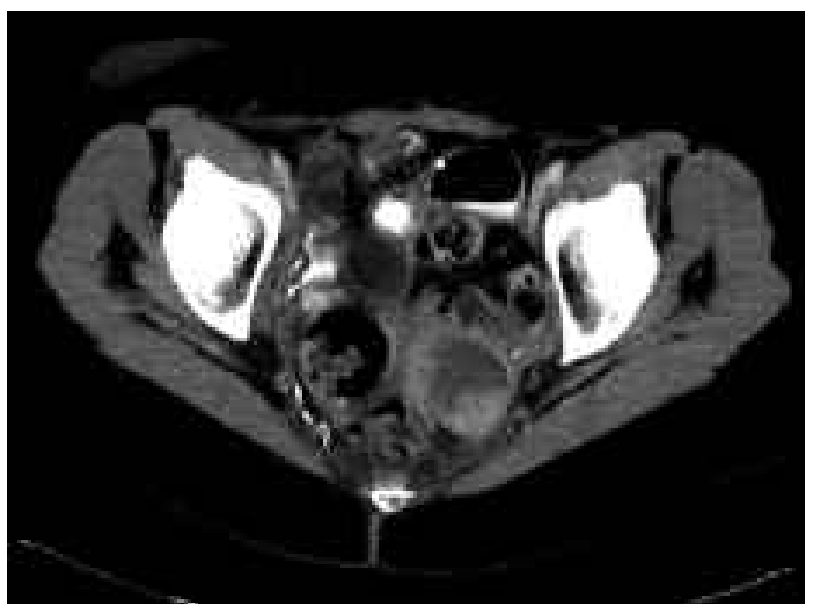

FIGURA 5. T.C. donde se visualiza la segunda recidiva a nivel de recto-sigma.

sarcomas vesicales más frecuentes son el sarcoma botrioide y el rabdomiosarcoma, que se presentan fundamentalmente en niños ${ }^{10,11}$. En el adulto, los sarcomas de vejiga son $\operatorname{raros}^{12}$, siendo el leiomiosarcoma el más común de todos los tumores mesenquimales malignos de la vejiga en el adulto y representa menos del $1 \%$ de los tumores vesicales 8 y entre el 13 y el 38\% de todos los sarcomas de la pared vesical $^{13}$. Desde que Gussembauer describió el primer caso de leiomiosarcoma en $1875^{14,15}$, no son muchos los casos publicados.

El leiomiosarcoma es un tumor derivado del músculo liso. Presenta un predominio por varones adultos, con una proporción $2: 1^{16}$. En cuanto a sus localización alrededor del 50\% se presenta en cúpula vesical y un $25 \%$ en las caras laterales. El síntoma predominante en casi todos los casos es la hematuria, aunque puede presentarse como un sindrome miccional irritativo, disuria, dolor abdominal ${ }^{14,17}$.

Parece que tanto los tumores que contienen estructuras presentes en la pared vesical (leiomiosarcoma y angiosarcoma) como los que presentan estructuras no presentes en la pared vesical (rabdomiosarcoma, liposarcoma, osteosarcoma, condrosarcoma), todos provienen de las células mesenquimales pluripotenciales de la pared vesical ${ }^{18}$.

A pesar de las numerosas hipótesis realizadas, la etiología es todavía desconocida. Se ha descrito la posible relación etiológica entre el tratamiento inmunosupresor con Ciclofosfamida ${ }^{18}$ y la aparición a los 10-15 años post-tratamiento de leiomiosarco$\mathrm{ma}^{16}$. La neurofibromatosis de Von Recklinghausen también se ha relacionado con la presencia de leiomiosarcomas ${ }^{18}$.
El diagnóstico se realiza con las técnicas habituales, aunque ninguna de ellas indica la presencia de leiomiosarcoma, por lo que el diagnóstico definitivo y el lugar primario de origen sólo puede realizarse mediante exploración quirúrgica y el subsecuente análisis histopatológico tras la escisión ${ }^{19}$.

La cistoscopia muestra una tumoración con forma de hongo, con áreas de desprendimiento central e incrustación cálcica ${ }^{15}$.

Es importante que las biopsias sean amplias y profundas, dado que las biopsias múltiples y superficiales son de muy baja sensibilidad en el diagnóstico anatomo-patológico del leiomiosarcoma ${ }^{16}$. Histológicamente se compone de células fusiformes ${ }^{15,18}$ con características anaplásicas y elevado número de mitosis ${ }^{14}$.

El diagnóstico diferencial se debe realizar con otros tumores vesicales con patrón fusocelular, como el leiomioma, rabdomiosarcoma, el carcinoma sarcomatoide, los nódulos proliferativos de células del huso y el pseudotumor inflamatorio ${ }^{14,16}$. Es esencial el diagnóstico diferencial de los pseudosarcomas inflamatorios, lesión que aparece como consecuencia de cirugías previas, sobretodo resecciones transuretrales, para evitar la realización de una cirugía cruenta innecesaria ${ }^{16,20}$.

El diagnóstico inmunohistoquímico confirma el origen muscular, siendo necesaria la utilización de métodos inmunohistoquímicos cuando se trata de tumoraciones muy indiferenciadas ${ }^{14,15,18}$. En estos, la presencia de desmina, vimentina y actina músculo específica confirma el diagnóstico de leiomiosarcoma, también se ha descrito la positividad a los antígenos epiteliales de membrana y a la citoqueratina ${ }^{18}$. Para algunos autores las tinciones inmunohistoquímicas son siempre necesarias ${ }^{20}$. La microscopia electrónica muestra por un prominente retículo endoplásmico rugoso y abundantes filamentos intracitoplasmáticos ${ }^{18}$.

La aplicación de técnicas de biología molecular, nos permiten diferenciar entre leiomiomas y leiomiosarcomas. Una de ellas, es la medición del contenido en DNA presente en el tumor. En el trabajo de Martin et $\mathrm{al}^{1}$. los leiomiomas presentaban un DNA diploide, mientras que los leiomiosarcomas presentaban un DNA tetraploide o aneuploide. Otra de las técnicas que nos permite realizar un diagnóstico diferencial con el leiomioma, es la actividad proliferativa del MIB-1, siendo esta significativamente más alta en los leiomiosarcomas que en los leiomiomas, aunque estos también expresan dicha actividad ${ }^{1}$. 
Aunque las características histológicas de los leiomiosarcomas vesicales son similares a las de tumores malignos del músculo liso con origen en otros lugares, los rasgos característicos para predecir su comportamiento y los criterios para diferenciar los leiomiosarcomas de los leiomiomas, no están bien establecidos.

Las dos series más amplias y con mayor seguimiento son las de Martin et al. ${ }^{1}$ y Mills et al. ${ }^{8}$ quienes proponen diferenciar entre leiomiosarcomas de alto y bajo riesgo en función del número de mitosis por 10 campos de gran aumento (CGA), de manera que si presentan menos de cinco mitosis por 10 CGA, se trata de un tumor de bajo riesgo, y si por el contrario, presentan más de cinco mitosis por 10 CGA, se consideran de alto riesgo. Presentando un comportamiento radicalmente opuesto, con una mediana de supervivencia de 7 meses para los de alto riesgo y de 65 meses para los de bajo riesgo. A su vez, refieren que el pronóstico de los tumores de bajo riesgo empeora cuando estos se encuentran infiltrando la muscular propia, aumentando el riesgo de metástasis y recidiva local. La particularidad del caso que presentamos, es que se trata de un tumor de alto riesgo, confinado a la capa muscular de la submucosa, con la capa muscular propia integra, su evolución ha sido tórpida, con una recidiva local al año y posteriores recidivas locales, sin evidenciar, lesiones metastáticas en ningún momento. También se ha estudiado el grado de necrosis tumoral, considerándose por algunos autores como el mejor parámetro pronóstico ${ }^{14,15}$.

Las metástasis suelen asentar en pulmón, hígado e intestino ${ }^{14,15}$. Aunque no existe un consenso claro respecto al tratamiento, se ha propuesto desde la resección endoscópica seguida de tratamiento con láser hasta la cistectomía ${ }^{14,18}$, con o sin tratamiento complementario. La resección completa es el único tratamiento eficaz ${ }^{15,20}$ probablemente por el potencial invasivo de estas lesiones, por lo que la cirugía radical continúa siendo el tratamiento de elección ${ }^{1,8,14}$. Algunos autores proponen la cistectomía parcial en aquellos casos en los que la localización tumoral permite una exéresis del tumor con márgenes quirúrgicos adecua$\operatorname{dos}^{18}$ de al menos $2 \mathrm{~cm}$ de tejido sano ${ }^{14}$. Otro planteamiento para realizar tratamiento conservador, son aquellos tumores de mínima actividad mitótica y ausencia de atipia celular, por ser tumores que raramente recidivan ${ }^{16}$.
Respecto a los tratamientos complementarios, bien neoadyuvante o adyuvantes, no hay mucho consenso y se reservan como parte de un tratamiento multimodal de leiomiosarcomas de alto grado $\mathrm{y}$ metastáticos. Se considera que no son radiosensibles ${ }^{10,14,15}$ por lo que sólo, en casos con enfermedad residual está indicada la radioterapia ${ }^{16}$ y la quimioterapia se reserva para los casos con diseminación sistémica aunque con pobres resultados. Se ha utilizado poliquimioterapia con Ciclofosfamida, Vincristina y Doxorrubicina ${ }^{14}$. Así como pautas de quimio-radioterapia neoadyuvante, con quimioterapia intraarterial con Cisplatino, Metrotexate y Pirarrubicin y radioterapia externa con $40 \mathrm{~Gy}$, para conseguir disminución de la masa tumoral previa a la cirugía ${ }^{21}$, e incluso se han realizado transplantes de medula ósea, como último esfuerzo terapéutico ${ }^{22}$.

Se han descrito casos de regresión espontánea de la enfermedad residual 5 meses después de finalizar un tratamiento con quimioterapia ${ }^{23}$ y sin evidencia de enfermedad 51 meses después del diagnóstico.

En general, el comportamiento de estos tumores es muy agresivo, con menos de un $40 \%$ de pacientes libres de tumor después de la resección. Todos los leiomiosarcomas de vejiga tienen potencial metastático, aunque los de alto riesgo presentan un comportamiento más agresivo ${ }^{1,8}$ con la supervivencia a 5 años del $63 \%{ }^{15}$.

El diagnóstico temprano, la cistectomía, radioterapia y quimioterapia, parecen mejorar el pronóstico ${ }^{24}$.

\section{CONCLUSIÓN}

Los leiomiosarcomas vesicales son tumores agresivos que se caracterizan por su recurrencia. Su diagnóstico diferencial con el leiomioma condiciona la actitud terapéutica, debiendo ser agresiva y multimodal por su agresivo comportamiento.

\section{REFERENCIAS}

1. Martin SA, Sears DL, Sebo TJ, Lohse CM, Cheville JC. Smooth muscle neoplasms of the urinary bladder: a clinicopathologic comparison of leiomyoma and leiomyosarcoma. Am J Surg Pathol. 2002 Mar;26(3):292-300.

2. AB C. Leiomyosarcoma of the urinary bladder: report of a case. J Urol 70:257,953.

3. Alabaster AM, Jordan WP Jr, Soloway MS, Shippel RM, Young JM.. Leiomyosarcoma of the bladder and subsequent urethral recurrence. J Urol. 1981 Apr;125(4):583-585.

4. Borzi P, Frank J. Bladder leiomyosarcoma in a child: a 6 year follow-up. Br J Urol. 1994 Feb;73(2):219-220. 
5. Chen K. Coexisting leiomyosarcoma and transitional cell carcinoma of the urinary bladder. J Surg Oncol. 1986 Sep;33(1):3637.

6. Cibert J, Durand L. Results of surgical treatment of 10 cases of myosarcoma of bladder. J Urol. 1954 Jan;71(1):58-62.

7. Hager B, Hunt V. A report of a case of leiomyosarcoma of the bladder. J Urol 1929,21.

8. Mills SE, Bova GS, Wick MR, Young RH.. Leiomyosarcoma of the urinary bladder: a clinicopathologic and immunohistochemical study of 15 cases. Am J Surg Pathol. 1989 Jun;13(6): 480-489.

9. Sanchez Gomez E. Bladder Leiomyosarcoma. Report of a new case. Actas Urol Esp. 1996 Oct;20(9):829-831.

10. Armas Molina JV, Chesa Ponce N, Martín Betancor D, Artiles Hernández JL, del Rosario Medina J, Ponce Socorro JM et al. Leiomiosarcoma vesical en joven trasplantado. Aportación de un caso. Actas Urol Esp. 1997 Mar;21(3):283-286.

11. Wu HY, Snyder HM 3rd. Pediatric urologic oncology: bladder, prostate, testis. Urol Clin North Am. 2004 Aug;31(3):619-27, XI.

12. Parekh DJ, Jung C, O'Conner J, Dutta S, Smith ER Jr. Leiomyosarcoma in urinary bladder after cyclophosphamide therapy for retinoblastoma and review of bladder sarcomas. Urology. 2002 Jul;60(1): 164.

13. Messing E. Urothelial tumors of the urinary tract, Eighth ed. Philadelphia: Saunders, pp. 2732-2765, 2002.

14. Gutiérrez Mínguez E, Espiga Santamaría J, Arroyo Muñoz JL, Velasco Oses A. Leiomiosarcoma de vejiga urinaria. Una neoformación muy poco frecuente. Actas Urol Esp. 1996 Jun;20 (6):574-576.

15. Begara Morillas F, Luengo Alpuente S, Salinas Casado J, Rapariz González M, Hernandez Villaverde A, Esteban Fuertes $\mathrm{M}$ et al. Leiomiosarcoma en el seno de un divertículo vesical. Actas Urol Esp. 1995 Apr; 19(4):337-340.
16. Sorinas Delgado N, Berné Manero JM, Bono Ariño A, García Prats MD, Sanz Vélez JI. Leiomiosarcoma de vejiga urinaria. Actas Urol Esp. 2001 May;25(5):385-387.

17. Ozteke O, Demirel A, Aydin NE, Memis L. Bladder leiomyosarcoma: report of three cases. Int Urol Nephrol, 24: 393, 1992.

18. Gil Fabra J, Fernández Fernández A, Gracía Marzo A, Soria Ruiz S, Otero Mauricio G. Leiomiosarcoma vesical. Presentación de dos casos. Arch Esp Urol. 1996 Jan-Feb;49(1): 59-62.

19. Ataus S, Yaycioglu O, Demirkesen O, Uygun N, Yalcin V, Solok V. Retrovesical Leiomyosarcoma. Int J Urol. 2000 Dec;7(12): 467-469.

20. Jaidane M, Slama A, Ben Sorba N, Yacoubi T, Ghammed S, Elleuch A et al. Bladder Leiomyosarcoma: report of a case. Ann Urol (Paris). 2000 Jun;34(3):179-183.

21. Namiki S, Hoshi S, Suzuki K. Retrovesical leiomyosarcoma responsive to preoperative chemoradiotherapy: a case report. Hinyokika Kiyo. 1997 Aug;43(8):589-592.

22. Russo P, Brady MS, Conlon K, Hajdu SI, Fair WR, Herr HW et al. Adult urological Sarcoma. J Urol. 1992 Apr;147(4):1032-6; discussion 1036-1037.

23. Lei KI, Gwi E, Ma L, Liang EY, Johnson PJ. 'Spontaneous' regression of advanced leiomyosarcoma of the urinary bladder. Oncology. 1997 Jan-Feb;54(1):19-22.

24. Ameur A, al Bouzidi A, Chtata H, Benomar S, Draoui D. Leiomyosarcoma of the bladder. A propos of a case. J Urol (Paris). 1996; 102(4): 180-182.

Correspondencia autor: Dr. Jorge Rioja Zuazu Departamento de Urología. Clínica Universitaria de Navarra. Av. Pio XII 36 - 31080 Pamplona. Tel.: 948255400

E-mail autor: jriojazu@gmail.com Información artículo: Nota clínica Trabajo recibido: septiembre 2007 Trabajo aceptado: octubre 2007 